\title{
Clasificación de pan francés según sus propiedades fisicoquímicas mediante la aplicación de los análisis de conglomerados y discriminante
}

\author{
Carlos A. Suca-Apaza ${ }^{1}$, Fernando Suca-Apaza ${ }^{2}$ y Diana C. Rojas ${ }^{3}$ \\ (1) Facultad de Química e Ingeniería Química, Departamento Académico de Procesos, Universidad Nacional Mayor de \\ San Marcos, Lima-Perú (correo-e: csucaa@unmsm.edu.pe) \\ (2) Facultad de Química e Ingeniería Química, Departamento Académico de Análisis y Diseño de Procesos, Universidad \\ Nacional Mayor de San Marcos, Lima-Perú (correo-e: fsucaa@unmsm.edu.pe) \\ (3) Facultad de Química e Ingeniería Química, Escuela Profesional de Ingeniería Agroindustrial, Universidad Nacional \\ Mayor de San Marcos, Lima-Perú (correo-e: diana.rojas5@unmsm.edu.pe)
}

${ }^{*}$ Autor a quien debe ser dirigida la correspondencia

Recibido Jul. 20, 2021; Aceptado Sep. 23, 2021; Versión final Nov. 8, 2021, Publicado Feb. 2022

\begin{abstract}
Resumen
El objetivo de esta investigación fue describir y clasificar el pan francés de panaderías de barrio y de supermercados de la Lima Metropolitana (Perú), mediante el análisis de conglomerados y discriminante. Se determinó la masa, volumen, volumen específico, humedad, acidez y contenido de sal de 198 muestras de pan Francés recolectadas de 66 panaderías por medio del muestreo de conveniencia. Usando los análisis multivariados, las muestras fueron clasificadas en tres grupos. Los mayores promedios de masa y volumen por unidad de pan correspondieron a los panes de supermercado; mientras que el volumen específico varió de 3.59 a $14.67 \mathrm{~cm}^{3} / \mathrm{g}$. Los panes de supermercado estuvieron dentro del parámetro de humedad dado por la normativa nacional peruana; en cambio, los de panaderías de barrio presentaron baja humedad y elevado volumen específico. Se concluye que el pan Francés en Lima Metropolitana puede ser clasificado en tres grupos según sus características fisicoquímicas.
\end{abstract}

Palabras clave: pan francés; volumen específico; análisis; conglomerado; discriminante

\section{Physicochemical classification of French bread by cluster and discriminant analyses}

\begin{abstract}
The aim of this research study was to describe and classify French bread from neighbourhood bakeries and grocery stores in the Lima Metropolitan Area (Peru) by cluster and discriminant analyses. Mass, volume, specific volume, moisture, acidity, and salt content of 198 French bread samples were collected from 66 bakeries selected by convenience sampling. Samples were classified into three groups by using multivariate analyses. The highest mass and volume averages per bread unit were found in grocery store French breads. Specific volume ranged from 3.59 to $14.67 \mathrm{~cm}^{3} / \mathrm{g}$. Grocery store breads were within humidity parameters set by Peruvian national regulations. However, neighbourhood bakery breads showed low humidity and high specific volume. It is concluded that French bread from the Lima Metropolitan Area can be classified into three groups according to their physicochemical characteristics.
\end{abstract}

Keywords: French bread; specific volume; cluster analysis; discriminant 


\section{INTRODUCCIÓN}

El pan es uno de los productos básicos más consumidos en el mundo. Se estima que el consumo per cápita de pan en el Perú es de $35 \mathrm{~kg} / \mathrm{año}$ (Sociedad Nacional de Industrias-SNI, 2018). Dentro de los panes tradicionales, el pan francés es una de las variedades de pan más producida y consumida en Lima Metropolitana. Se le puede encontrar en casi todas las panaderías y la razón de su popularidad probablemente estriba en su palatabilidad y asequibilidad económica. Según la Norma Técnica Peruana, NTP 106.004 (2016), al pan francés se le define como un pan tradicional a base de harina de trigo, azúcar, sal, fermento y agua, con o sin contenido de grasa y mejoradores de masa. En cuanto a las características físicas, esta misma norma menciona que "el pan francés tiene forma ovalada, con una hendidura sobre el eje longitudinal de la cara superior, corteza crujiente y dorada, miga blanca-cremosa y esponjosa, y sabor característico". Estas propiedades pueden modificarse, no obstante, como consecuencia de los cambios en la formulación y los procesos de elaboración, resultando en productos con características ampliamente variables (Mondal y Datta, 2008; Gerardo-Rodríguez et al., 2021).

La calidad del pan francés puede ser evaluada mediante análisis físicos, químicos y microbiológicos. Según la NTP 106.004 (2016), el pan francés debe tener una humedad mínima de $25 \%$, y un máximo de $35 \%$; dos por ciento de sal como máximo y $3 \%$ de grasa como máximo. Esta misma norma, establece tres categorías de panes francés: gourmet, comercial y sánguche. En relación al peso, los panes gourmet deben pesar entre $8 \mathrm{~g}$ a $15 \mathrm{~g}$; mientras que el pan francés comercial deberá tener entre 30 y $40 \mathrm{~g}$; en tanto que la categoría sánguche puede ser mayor a $40 \mathrm{~g}$. En cuanto a la acidez, los panes elaborados con harina de trigo tendrán un máximo de $0.4 \%$ de acidez (expresado en ácido sulfúrico); mientras que las mezclas con harinas sucedáneas no deberán exceder de $0.5 \%$.

Dentro de las propiedades físicas del pan, el volumen es una de las más importantes (Rossmann et al., 2020). El volumen de un pan puede variar a causa de varios factores, como por ejemplo la adición de aditivos que afecten el comportamiento de los lípidos (Melis et al., 2019), y el uso de cepas de $S$. cerevisiae que no son específicos para su uso en panadería (Laurent et al., 2020). Otro parámetro importante en el análisis de los panes es el volumen específico, según Alvis et al. (2011) y Mudgil et al. (2016). Por ello, dada la creciente exigencia de los consumidores, el control de la variabilidad en la producción debe ser una prioridad para las empresas que desean mantener la calidad de sus productos y, por tanto, sus cuotas en el mercado.

Por otro lado, las técnicas estadísticas multivariadas son herramientas muy poderosas y avanzadas que permiten revelar una matriz compleja de datos (da Silva Torres et al., 2006; Mohammadi-Moghaddam y Firoozzare, 2021). Estas herramientas son aplicables en contextos de investigación muy diferentes; y su uso e interpretación dependerán precisamente del contexto en el que se les aplique. Cuando el contexto requiere clasificar individuos o variables que cuenten con características semejantes, sin tener a priori un criterio de clasificación, se opta por el uso del análisis de conglomerados y el uso de métodos de acuerdo a la distancia de los datos procesados. Así lo confirman Cardone et al. (2020), al clasificar panes fabricados con harinas de trigo germinados desde 24 a 62 h; y da Silva Torres et al. (2006) al emplearlas en estudios de composición de alimentos. A los estudios anteriores adicionamos los llevados a cabo por Mohammadi-Moghaddam y Firoozzare (2021), y Yousaf et al. (2021), quienes emplearon el análisis discriminante y de conglomerados, respectivamente; aplicados en la aceptación de mermeladas de ciruela negra como efecto de sus propiedades sensoriales; y la caracterización fisicoquímica y nutracéutica de cultivares de guayabas.

A partir de una matriz de individuos-variables, el análisis de conglomerados trata de situar los casos (individuos o variables) en grupos homogéneos no conocidos de antemano, pero sugeridos por la propia esencia de los datos (Pérez, 2013). Se cuenta con dos métodos diferentes para agrupar a los sujetos más similares entre sí y que los grupos sean lo más diferentes posible entre sí: a) métodos jerárquicos y b) métodos no jerárquicos. Mediante el uso de métodos jerárquicos se construye un árbol de clasificación denominado dendograma el cual permite mostrar los grupos que se unen, así como el nivel en que lo hacen. Dentro de los métodos jerárquicos, el método de Ward toma en cuenta a cada individuo en un grupo independiente y así, va agrupado a los más cercanos hasta formar un grupo homogéneo.

De acuerdo con De La Hoz y Polo (2017), la última etapa de la metodología consiste en formar un perfil de los conglomerados que funcionará como una muestra para comparar los factores entre un conglomerado y otro; así también evalúan si las variables establecidas anteriormente son útiles para una adecuada discriminación. Por todo lo expuesto, el presente trabajo tiene por objetivo describir y clasificar muestras de panes francés procedentes de panaderías ubicadas en Lima Metropolitana para categorizarlos empleando las técnicas multivariadas de análisis de conglomerados y análisis discriminante. Los resultados podrán emplearse en la mejora de los procesos, en la revisión y actualización de normas técnicas que redundarán en la mejora de la uniformidad y calidad de los panes producidos. 


\section{METODOLOGIA}

El presente trabajo comprendió las etapas de la recolección de muestras, preparación y medición de las propiedades físicas y químicas, así como el análisis de los datos obtenidos. Por otro lado, cabe mencionar que los autores no poseen conflicto de interés con las empresas a las que se hace mención.

\section{Recolección de muestras}

Para iniciar con la recolección de las muestras, las panaderías de donde se recolectaron las mismas fueron categorizadas en dos grupos: a) panaderías de barrio y b) panaderías de supermercado. Se recogieron muestras de tres panaderías de barrio de cada uno de los siguientes distritos de Lima Metropolitana: Puente Piedra, Comas, Los Olivos, San Martín de Porres, Villa El Salvador, Chorrillos, Villa María del Triunfo, Barranco, San Juan de Lurigancho, La Victoria, Breña, Rímac y Lima centro. En cambio, con respecto a la toma de muestras de las panaderías de supermercado, éstas se subcategorizaron según la empresa a la cual pertenecen. De esa manera, se tuvo muestras de las panaderías ubicadas al interior de las tiendas pertenecientes al grupo Cencosud (Metro y Wong), grupo Intercorp (Plaza Vea) y grupo Falabella (Tottus); las mismas que se encuentran en diferentes distritos de Lima Metropolitana.

Se obtuvieron muestras de 48 panaderías de barrio, siete panaderías dentro de los supermercados del grupo Cencosud, cinco panaderías dentro de las tiendas del grupo Intercorp y seis panaderías de las tiendas del grupo Falabella; llegando a un total de 66 panaderías. Como era prácticamente imposible realizar un muestreo aleatorio de las panaderías de barrio, que aportarían con las muestras para el estudio, se optó por realizar un muestreo de conveniencia, es decir, la muestra se tomó cuando surgía la oportunidad de encontrar una panadería en los distritos mencionados. Se recogieron tres panes francés de cada una de las panaderías mencionadas, totalizando 198 unidades. El recojo de las muestras de panaderías de barrio se realizó por la mañana, de 6:00 hasta las 8:00; mientras que las muestras de panaderías de supermercado se recogieron entre las 14:00 a 16:00 horas. De esta manera se logró que los panes fueran recogidos y analizados dentro de las dos horas posteriores a su elaboración, conforme a lo señalado por Rodrigues Ferreira et al. (2001) y las directrices dadas en la NTP 206.006 (2016). Los panes fueron cuidadosamente embolsados, codificados y trasladados en cajas de cartón corrugado para evitar magulladuras o deformaciones que pudieran alterar cualquiera de las propiedades físicas medidas, durante el transporte al laboratorio. El recojo se efectuó en varios días, de preferencia entre jueves y viernes, visitando hasta tres distritos por día.

\section{Preparación de las muestras}

Al llegar al laboratorio, cada pan recibió un código de tres letras y dos números para identificarlos y rastrearlos durante todo el análisis. Las letras hacían mención al distrito o supermercado del que provenían; y los números, a la panadería y al pan. Una vez codificadas, las muestras fueron colocadas en platillos descartables.

\section{Medición de las propiedades físicas}

Las propiedades físicas analizadas al pan fueron: masa unitaria $(\mathrm{g})$, volumen unitario $\left(\mathrm{cm}^{3}\right)$, densidad $\left(\mathrm{g} / \mathrm{cm}^{3}\right)$ y volumen específico $\left(\mathrm{cm}^{3} / \mathrm{g}\right)$. La masa de cada pan se determinó en una balanza digital con aproximación de centésimas de gramo, inmediatamente después de recibir la codificación. Luego, se obtuvo el volumen correspondiente utilizando el método de desplazamiento de semillas de la AACC International Method 1005.01 (2001); con la diferencia de que en lugar de usar semillas de colza se utilizaron semillas de chía (Salvia hispanica L.), con una humedad de $(7.0 \pm 0.4) \%$, y una densidad volumétrica de $(0.6546 \pm 0.0005) \mathrm{g} / \mathrm{cm}^{3}$. En un cubo de material acrílico de $2000 \mathrm{~cm}^{3}$, se dejó caer entre $150 \mathrm{~g} \mathrm{a} 200 \mathrm{~g}$ de semillas de chía desde una altura de $15 \mathrm{~cm}$ con respecto al borde superior de dicho cubo; esto con la finalidad de servir como asiento para la muestra, ya que ésta suele tener una base cóncava que puede generar un error en la lectura del volumen del pan. Una vez que la muestra fue colocada, se terminó de rellenar el cubo con más semillas de chía, hasta completar el espacio faltante y desbordando la capacidad del mismo. Se enrasó con una regla y se procedió a pesar el cubo con el contenido descrito. El volumen del pan se determinó calculando el volumen de granos de chía que no ingresó al cubo debido a que la muestra ocupó su lugar. Dicho volumen se calculó empleando la densidad volumétrica de los granos de chía dada más arriba, cuyo valor se determinó restando la masa del cubo lleno de semillas menos la masa del cubo con la muestra. Con la masa del pan y su volumen se calculó su densidad y el volumen específico de cada pan se obtuvo a partir del recíproco de su correspondiente densidad.

\section{Determinación de humedad, sal y acidez}

Los panes enteros debidamente identificados fueron llevados a una estufa a $100{ }^{\circ} \mathrm{C}$ durante $24 \mathrm{~h}$ para determinar su humedad. Se obtuvo la cantidad de agua presente por diferencia entre la masa inicial y la masa final después de la determinación, y dividiendo dicha cantidad entre la masa inicial. Este valor se expresó 
como porcentaje. Los panes secos enteros fueron molidos con ayuda de un molino manual y envasados en bolsitas de polietileno hasta el momento de llevar a cabo los análisis de contenido de sal y acidez. La determinación del contenido de sal se efectuó mediante el método de Mohr, dado en la AOAC Method 983.14 (2005), utilizando una solución estandarizada de $\mathrm{AgNO}_{3} 0.1 \mathrm{~N}$, y siguiendo los lineamientos de dicho método. Se reportó el promedio de contenido para cada panadería. En cuanto a la determinación de acidez, se procedió a través del método de titulación con una solución estandarizada de $\mathrm{NaOH} 0.05 \mathrm{~N}$ según la NTP 206.008 (2016). Se pesó $25 \mathrm{~g}$ de pan seco y en polvo, el cual se diluyó en agua destilada. La solución fue filtrada para proceder a la titulación. La acidez se expresó como porcentaje de ácido sulfúrico, con una equivalencia de $1 \mathrm{~mL}$ de $\mathrm{NaOH} 0.05 \mathrm{~N}$ gastado en la titulación igual a $0.0024 \mathrm{~g}$ de ácido sulfúrico.

\section{Análisis estadísticos de los datos}

Las herramientas utilizadas para clasificar las muestras de pan, así como verificar la idoneidad de los conglomerados formados fueron el análisis de conglomerados y el análisis discriminante. Se ha optado por estas técnicas ya que permiten clasificar los panes en grupos homogéneos, en donde poco o nada se sabe sobre ellos antes de ser clasificados, lo que representa una ventaja; puesto que no se condiciona a priori la pertenencia de una muestra a un grupo. Su carácter multivariado permite, además, que la clasificación se haga considerando simultáneamente la contribución de cada una de las variables estudiadas en los panes. Dichas variables fueron: masa, volumen, volumen específico y humedad. El método de conglomeración fue el de Ward utilizando como métrica de distancia la euclideana cuadrada. Los resultados fueron evaluados utilizando el paquete estadístico Statgraphics Centurion XVII (Statpoint Technologies; VA, USA, 2014). Luego, el análisis discriminante permitió verificar la pertinencia de los datos a los conglomerados obtenidos. También se obtuvieron las estadísticas descriptivas usando este mismo paquete estadístico.

\section{RESULTADOS}

Los resultados del análisis de conglomerados por el método de Ward, para efectos prácticos fueron clasificados en tres conglomerados: CON1, CON2 y CON3, respectivamente. El $100 \%$ de los panes que integran el CON1 proviene de las panaderías de barrio, estando conformado por 105 panes de los 198 recogidos. Para el CON2, el $90 \%$ lo integran las muestras que provienen de panaderías de barrio, mientras que el $10 \%$ restante proviene de las panaderías del supermercado Tottus. Este segundo conglomerado está conformado por 40 muestras de las 198 recogidas. En cambio, 50 de los 53 panes que conforman el CON3 provienen de las panaderías de supermercado. El CON1 representa aproximadamente el $53 \%$ del total de las muestras recogidas, mientras que el CON2, el $20 \%$; y el CON3, el restante $27 \%$.

Pérez (2013) indica que después de llevar a cabo un análisis de conglomerados, la etapa final es hacer un perfil de los conglomerados obtenidos realizando un análisis discriminante. Según esto, de los 105 panes que integran el CON1, noventa y siete panes fueron correctamente agrupados en este conglomerado, mientras que los restantes ocho panes fueron agrupados en el CON2. Igualmente, de los 40 panes agrupados en el CON2, treinta y ocho fueron correctamente agrupados en el CON2, y los restantes dos panes fueron agrupados en el CON1 y CON3, uno en cada conglomerado. Finalmente, cincuenta de los 53 panes del CON3 fueron correctamente asignados a este conglomerado, mientras que los tres restantes quedaron fijados en el CON2.

El análisis discriminante también reporta dos funciones discriminantes para los tres grupos formados por conglomeración. La primera función es capaz de discriminar en un $92.4 \%$ de probabilidad. Debido a su alta capacidad discriminativa, la ecuación (1) pertenece a la primera función discriminante estandarizada; donde se observa que los coeficientes que más influyen en la capacidad discriminante son las variables volumen del pan $\left(V_{p}\right)$ y volumen específico del pan $\left(V_{p}\right)$.

$$
\operatorname{CON}_{i}=\left(0,26 \times m_{p}\right)+\left(0,85 \times V_{p}\right)-\left(0,91 \times v_{p}\right)+\left(0,07 \times h_{p}\right)
$$

En cambio, la segunda función discriminante (ecuación no mostrada) es capaz de discriminar los resultados en un $7.6 \%$. La Tabla 1 presenta las estadísticas descriptivas de los conglomerados según las variables analizadas luego de someter los datos a un análisis discriminante. Se tiene en cuenta que las letras iguales en la misma fila significan similitud estadística al 1\% entre conglomerados; y los conglomerados que son estadísticamente diferentes en cada una de las variables estudiadas $(p<0,01)$ se muestran con el símbolo $\left.{ }^{* *}\right)$. Los coeficientes de variabilidad (CV) para la masa de los panes dentro del CON2 y CON3 superan el $10 \%$.

Para el CON1, el CV de la masa individual de las muestras es menor a 10\%, por lo que podría decirse que existe una variabilidad aceptable de la masa del pan dentro de este conglomerado. En estudios exploratorios como éste, donde ninguna de las variables estudiadas ha sido controlada por los investigadores, se espera valores altos de CV (mayores a 10\%), tal como lo que ocurre con el CV del volumen específico en el CON3, 
que supera el $20 \%$. A esta variabilidad habrían contribuido los factores durante el proceso de producción, diferencias en las proporciones de los ingredientes, distintos tiempos de horneado, así como técnicas variadas de fabricación de los panes; todos ellos propios de cada panadería. Para estudios de naturaleza experimental llevados a cabo en laboratorios, los valores aceptables de CV serían de no más de 5\%, ya que las variables son mucho más controladas por los experimentadores. Por lo mencionado, los CV altos obtenidos están justificados por ser éstos características propias de un muestreo de panes de diferentes panaderías.

Tabla 1: Propiedades de los panes francés caracterizados por conglomerados.

\begin{tabular}{|l|c|c|c|c|c|c|c|c|c|c|}
\hline & \multirow{2}{*}{ Razón F } & \multicolumn{3}{|c|}{ Conglomerado 1 } & \multicolumn{3}{c|}{ Conglomerado 2 } & \multicolumn{3}{c|}{ Conglomerado 3 } \\
\cline { 3 - 11 } & Media & DS & CV (\%) & Media & DS & CV (\%) & Media & DS & CV (\%) \\
\hline $\begin{array}{l}\text { Masa del } \\
\text { pan (g) }\end{array}$ & $557,6^{* *}$ & $28,1 \pm 0,5^{\mathrm{a}}$ & 2,7 & 9,6 & $36,0 \pm 1,8^{\mathrm{b}}$ & 5,8 & 15,9 & $63,5 \pm 2,8^{\mathrm{c}}$ & 10,5 & 16,6 \\
\hline $\begin{array}{l}\text { Volumen } \\
\text { del pan } \\
\left(\mathrm{cm}^{3}\right)\end{array}$ & $75,0^{* *}$ & $279,0 \pm 7,3^{\mathrm{a}}$ & 38,3 & 13,7 & $266,3 \pm 6,2^{\mathrm{a}}$ & 19,9 & 7,5 & $343,3 \pm 9,9^{\mathrm{b}}$ & 36,7 & 10,7 \\
\hline $\begin{array}{l}\text { Volumen } \\
\text { específico } \\
\text { del pan } \\
\left(\mathrm{cm}^{3} / \mathrm{g}\right)\end{array}$ & $206,6^{* *}$ & $9,9 \pm 0,3^{\mathrm{a}}$ & 1,4 & 14,3 & $7,5 \pm 0,3^{\mathrm{b}}$ & 1,0 & 13,7 & $5,6 \pm 0,3^{\mathrm{c}}$ & 1,3 & 22,8 \\
\hline $\begin{array}{l}\text { Humedad } \\
(\%)\end{array}$ & $118,5^{* *}$ & $19,7 \pm 0,4^{\mathrm{a}}$ & 2,3 & 11,5 & $24,3 \pm 0,7^{\mathrm{b}}$ & 2,3 & 9,5 & $26,1 \pm 1,0^{\mathrm{c}}$ & 3,5 & 13,5 \\
\hline
\end{tabular}

En la Tabla 1, también se observa que el CON3 reúne a los panes con el mayor promedio de masa, seguido de los panes del CON2; mientras que, el CON1 presenta el promedio más bajo de masa de pan, además de tener el menor coeficiente de variabilidad de los tres en esta característica. Según la tabla de pesos comerciales del pan francés dada en la norma NTP 106.004 (2016), los panes del CON2 caen en la categoría de panes comerciales. No obstante, no se recolectaron las otras dos categorías de panes estipuladas en dicha norma. Los pesos adecuados del pan francés se rigen de acuerdo a las normas de cada país; así, Ortolan et al. (2015) al desarrollar un nuevo proceso y con mezclas de harinas para la elaboración del pan francés cumplieron con los parámetros establecidos de la norma brasileña NBR 16170, con pesos promedios de 53,82 y $50,67 \mathrm{~g}$.

Sin embargo, la masa de los panes analizados en este estudio varía entre panaderías e incluso entre muestras de una misma panadería. La variabilidad en las masas de los panes no perjudica económicamente a las panaderías de supermercado, puesto que éstas comercializan el pan por peso; sin embargo, las panaderías de barrio deberían buscar minimizar dicha variabilidad ya que la venta del pan es por unidad. Este hecho se ve reflejado cuando se compara los CV de la masa de los panes dentro de cada conglomerado (Tabla 1). Los promedios de los volúmenes del pan francés en los diferentes conglomerados variaron desde $271.67 \mathrm{~cm}^{3} \mathrm{a}$ $353.22 \mathrm{~cm}^{3}$ (Tabla 1), siendo el CON2 el que posee la menor variabilidad. En el CON1, el menor volumen de pan fue de $198 \mathrm{~cm}^{3}$ (dato no presentado), mientras que el mayor tuvo $381 \mathrm{~cm}^{3}$. De la misma forma, los volúmenes mínimo y máximo de los panes en el CON2 fueron de $213 \mathrm{~cm}^{3}$ y $320 \mathrm{~cm}^{3}$, respectivamente. En el CON3, el volumen mínimo del pan fue de $275 \mathrm{~cm}^{3}$, mientras que el máximo fue de $430 \mathrm{~cm}^{3}$. Rodrigues Ferreira et al. (2001) analizaron panes francés comerciales encontrando volúmenes entre $303 \mathrm{~cm}^{3}$ a $507 \mathrm{~cm}^{3}$.

En la Tabla 1 se observa que el volumen del pan es el que posee la menor razón $F$, lo que explica que es la propiedad que menos variabilidad presenta en forma global con respecto a las otras tres propiedades evaluadas; aunque estadísticamente exista diferencia significativa en los volúmenes del pan entre los conglomerados. Observando en forma global, esto también quiere decir que los volúmenes de los panes fueron bastante similares, dentro de la heterogeneidad observada. Los volúmenes mayores de pan pueden deberse a una fermentación promovida por las levaduras (Parenti et al. 2020), poca sal, harina con bajo contenido de gluten, gluten muy fuerte o débil, harina con bajo contenido de maltosa o baja hidratación de masa (Rodrigues Ferreira et al., 2001). Un volumen excesivo también puede ser causado por una cantidad excesiva de aditivos mejoradores, sales (Gusmão et al., 2016) o altas temperaturas de horneado.

El promedio del volumen específico más bajo lo tiene el CON3; mientras que el CON1 tiene el valor más alto en esta variable (Tabla 1). En las normas técnicas peruanas no se hace referencia al volumen específico como criterio de calidad en la evaluación de los panes. Para Rodrigues Ferreira et al. (2001) y Mudgil et al. (2016), el volumen específico es la variable más utilizada en la evaluación física de los panes, inclusive hay un intervalo de $5 \mathrm{~cm}^{3} / \mathrm{g}$ a $8 \mathrm{~cm}^{3} / \mathrm{g}$, que sería considerado adecuado para el pan francés. Según esto, solo los panes del CON2 y CON3 cumplirían con este parámetro. El rango de valores del volumen específico $\left(\mathrm{cm}^{3} / \mathrm{g}\right)$ para los conglomerados (datos no presentados) fueron, respectivamente: 7.32 a 14.67 para el CON1; 4.38 a 9.24 para el CON2, y 3.59 a 10.05 para el CON3. Únicamente el $5 \%$ de las muestras que conforman el CON1 
está dentro de este rango. Gusmão et al. (2017), constataron que la adición de aditivos alimentarios como el cloruro de potasio podrían, además de disminuir el uso de cloruro de sodio, mejorar las características fisicoquímicas como el volumen específico dentro de los valores indicados por Mudgil et al. (2016). Por su parte, Svec y Hrusková (2010) obtuvieron volúmenes específicos de pan desde 2,87 a 3,66 cm3/g al evaluar las características de harinas para panificación.

Por otro lado, compuestos como las puroindolinas en interacción con los lípidos podrían influir en el volumen específico del pan por lo que se requiere más estudios para confirmar ello (Melis et al., 2019). Además, el uso de ingredientes como polisacáridos junto a procesos de congelado de la masa también incrementarían el volumen específico de panes (Mudgil et al., 2016). Por todo ello, se podría afirmar que casi todas las panaderías utilizarían mejoradores u otros ingredientes que actúen sobre el gluten y enzimas para favorecer la producción de sustratos y, con éstos, incrementar el nivel de fermentación a fin de modificar los espacios de aire en el interior del pan.

La humedad de los panes del CON3 es la más alta de los tres conglomerados (Tabla 1), y solo el $13 \%$ de las muestras de este conglomerado supera el 30\% de humedad. La NTP 106.004 (2016) estipula un contenido mínimo de humedad de $25 \%$ para el pan francés, con un máximo de $35 \%$. Los panes del CON1 y CON2 no cumplirían con este requisito. Sin embargo, el incumplimiento en relación a la humedad no acarrea riesgo para los consumidores, aunque las humedades altas aumentan la probabilidad de contaminación y proliferación microbiana. No obstante, se debe mencionar que el pan francés es un producto de consumo inmediato, en fresco; por lo que valores de humedad por encima de lo estipulado no representarían riesgo sanitario para el consumidor (Rodrigues Ferreira et al., 2001).

En la Tabla 2 se presentan los resultados de las variables agrupadas por tipo de panadería (panadería de barrio y panadería de supermercado) y las variables en forma global. Se observa que los CV de todas las variables para panaderías de barrio y de supermercado están entre $10 \%$ a $20 \%$, indicando que están dentro de un rango de variabilidad acorde con la naturaleza de las variables estudiadas. Sin embargo, si se observa los datos en forma global, el mayor CV es para la masa de los panes, con $41.67 \%$ de variabilidad, lo que quiere decir que la masa es la variable más heterogénea estudiada en las muestras. En contraposición, el menor valor de CV para el volumen del pan indica que casi todas las muestras presentaron un volumen bastante similar. Por otro lado, los valores de las diferentes variables para las panaderías de supermercado son muy similares a los valores del CON3. Esto se debe a que casi la totalidad de las muestras (94 \%) de este conglomerado provienen de panaderías de supermercado, donde existe una mayor estandarización y homogenización en la producción de este alimento.

Tabla 2: Propiedades de panes francés caracterizados por tipo de panadería y en forma global.

\begin{tabular}{|l|c|c|c|c|c|c|c|c|c|}
\hline & \multicolumn{4}{|c|}{ Panaderías de barrio } & \multicolumn{3}{c|}{$\begin{array}{c}\text { Panaderías } \\
\text { de supermercado }\end{array}$} & \multicolumn{3}{c|}{ Panaderías en forma global } \\
\hline & Media & DS & CV (\%) & Media & DS & CV (\%) & Media & DS & CV (\%) \\
\hline Masa del pan (g) & $30,0 \pm 0,7$ & 4,5 & 15,1 & $63,6 \pm 2,7$ & 10,0 & 15,7 & $39,2 \pm 2,3$ & 16,3 & 41,7 \\
\hline Volumen del pan $\left(\mathrm{cm}^{3}\right)$ & $278,5 \pm 6,2$ & 37,6 & 13,5 & $334,2 \pm 11,3$ & 42,7 & 12,8 & $293,7 \pm 6,4$ & 46,3 & 15,8 \\
\hline $\begin{array}{l}\text { Volumen específico } \\
\text { del pan (cm } / \mathrm{g})\end{array}$ & $9,4 \pm 0,3$ & 1,6 & 16,8 & $5,3 \pm 0,2$ & 0,8 & 15,4 & $8,3 \pm 0,3$ & 2,3 & 27,8 \\
\hline Humedad (\%) & $20,9 \pm 0,5$ & 3,1 & 14,8 & $26,0 \pm 1,0$ & 3,6 & 13,7 & $22,3 \pm 0,6$ & 3,9 & 17,6 \\
\hline
\end{tabular}

En la Figura 1 se observa que el contenido de sal de las muestras varía en el rango de $1,2 \%$ a 2,8 \%. El promedio del contenido de sal para las muestras provenientes de panaderías de barrio fue de $(1.84 \pm 0.07) \%$, con un CV igual a $24,25 \%$; mientras que, para los panes de panaderías de supermercado, el promedio fue de $(2,28 \pm 0,07) \%$, con CV igual a 11,40\%. Posiblemente, la menor variabilidad del contenido de sal de las muestras de panaderías de supermercado se deba a la estandarización de las recetas industriales, al tratarse de panaderías con procesos productivos más normalizados que las panaderías de barrio. Por otro lado, se observa que los panes de supermercado tienen un contenido porcentual de sal mayor que lo estipulado en la NTP 106.004 (2016); a diferencia de los panes de panaderías de barrio que, en promedio, sí cumplen con la norma mencionada ( $2 \%$ máximo de contenido de sal).

El contenido de sal en el pan puede conllevar a diversos problemas de salud; cuyo límite máximo está regulado por las autoridades sanitarias de cada país. Gusmão et al. (2017) manifiesta que según la Guía de Buenas Prácticas de Nutrición para pan francés de la Agencia Nacional de Investigación en Salud del Brasil (ANVISA), se redujo progresivamente el contenido de sal de $2 \%$ a $1.8 \%$. La cantidad de sal puede ser reducida mediante el reemplazo de la sal común por cloruro de potasio entre un 0.2 y $0.5 \%$ sin observarse modificaciones significativas en los parámetros de calidad del pan francés (Antúnez et al., 2017); ya que la 
sal influye en comportamiento del gluten, refuerza la estructura de la masa, disminuye la producción de gas y la actividad de la levadura, mejorando el sabor del producto (Gusmão et al., 2017).

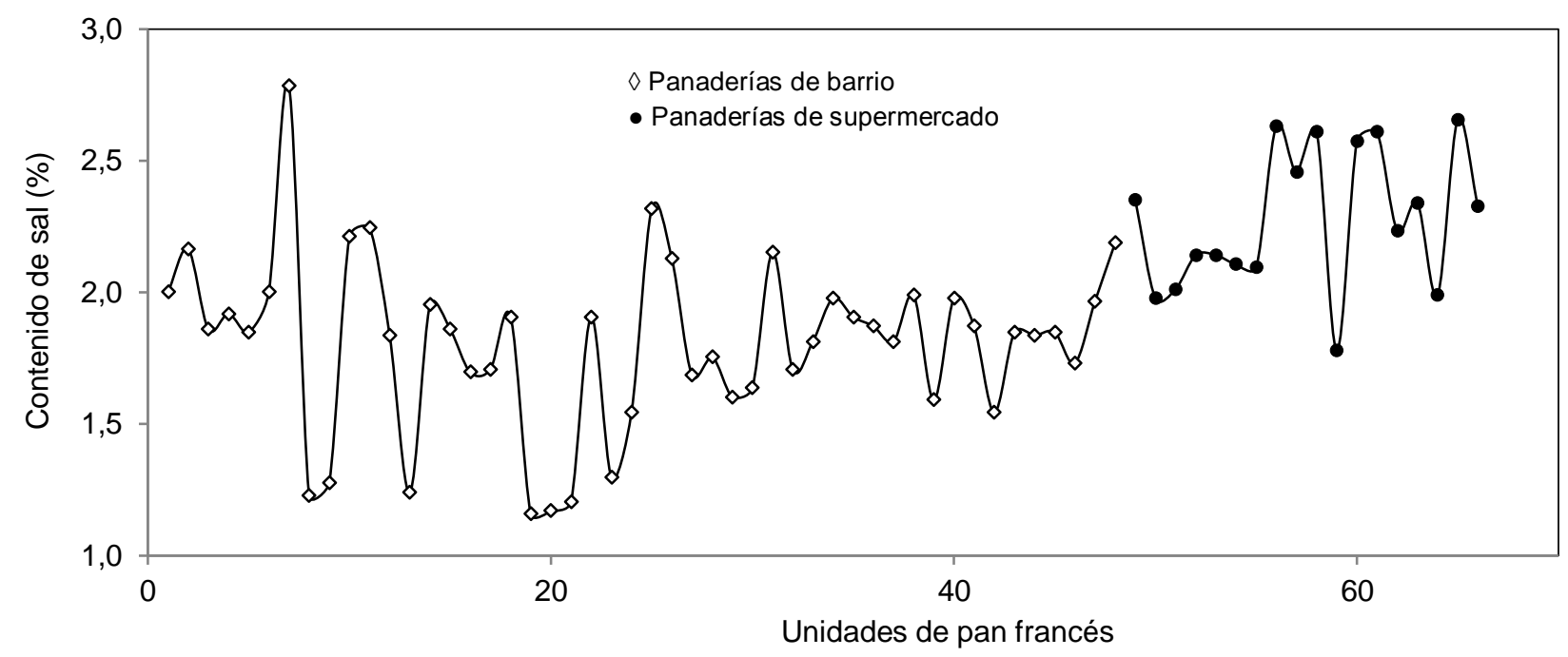

Fig. 1: Contenido de sal en panes francés

La Figura 2 muestra la variación de la acidez de los panes francés, el cual es similar entre los dos grupos de panaderías. Ambos promedios de acidez son estadísticamente iguales $(0.09 \%)$, aunque las muestras de panaderías de supermercado tienen mayor CV $(20,37 \%)$ que las de panaderías de barrio $(17,74 \%)$. Aunque la NTP 106.004 (2016) no especifique un límite máximo de porcentaje de acidez, menciona que la masa antes del horneado no debe presentar signos de putrefacción y; luego del horneado, la miga del pan no debe contar con zonas almidonosas y la corteza debe presentar un color dorado homogéneo. El control de las características sensoriales del pan brinda una señal de qué tan adecuada fue su fermentación. Si el proceso de fermentación se da a altas temperaturas y/o con largos periodos de tiempo, es muy probable que la acidez del pan se incremente debido al exceso de ácido láctico formado durante la fermentación (He y Lu, 2015). Por el contrario, estos mismos autores mencionan que si la fermentación se da a bajas temperaturas y por corto tiempo, se puede ver efectos negativos en el sabor y otras propiedades sensoriales del pan.

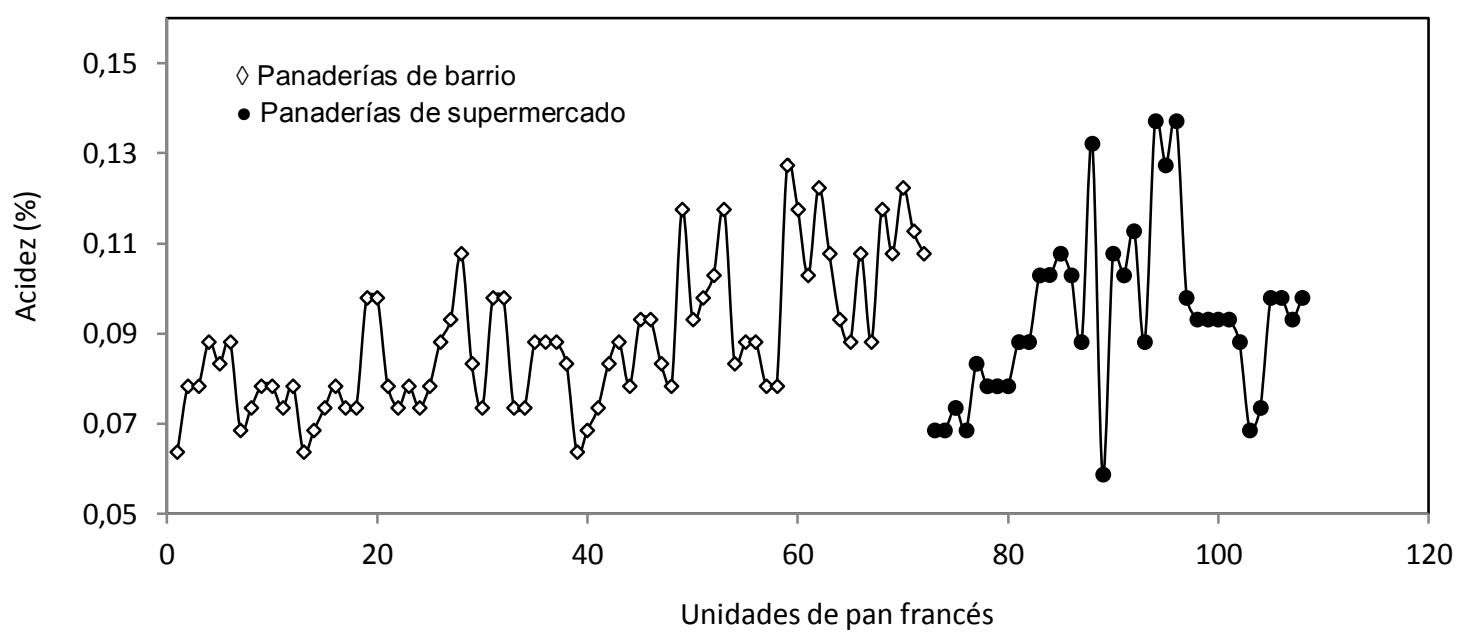

Fig. 2: Porcentaje de acidez (expresado en ácido sulfúrico) en panes francés

La humedad de los panes francés varía desde $12.03 \%$ a $31.60 \%$ (Figura 3). Se observa igualmente que el promedio de humedad de los panes es mayor para las panaderías de supermercado que para las panaderías de barrio. Esto se corrobora en la Tabla 2, en donde la humedad promedio de los panes de panaderías de supermercado es aproximadamente $5 \%$ mayor que el promedio de la humedad de los panes de panaderías de barrio. Los panes de supermercado se ajustan más a los parámetros de humedad de la NTP 106.004 (2016), la cual indica que el pan francés debe contar entre un 25 y $35 \%$ de humedad. Estos valores se asemejan a los hallados por Gusmão et al. (2017) quienes obtuvieron humedades de 29 y $31 \%$. Cabe mencionar que la humedad de los panes no debe exceder el estipulado en la norma ya que es uno de los factores que determina la vida útil del pan. 


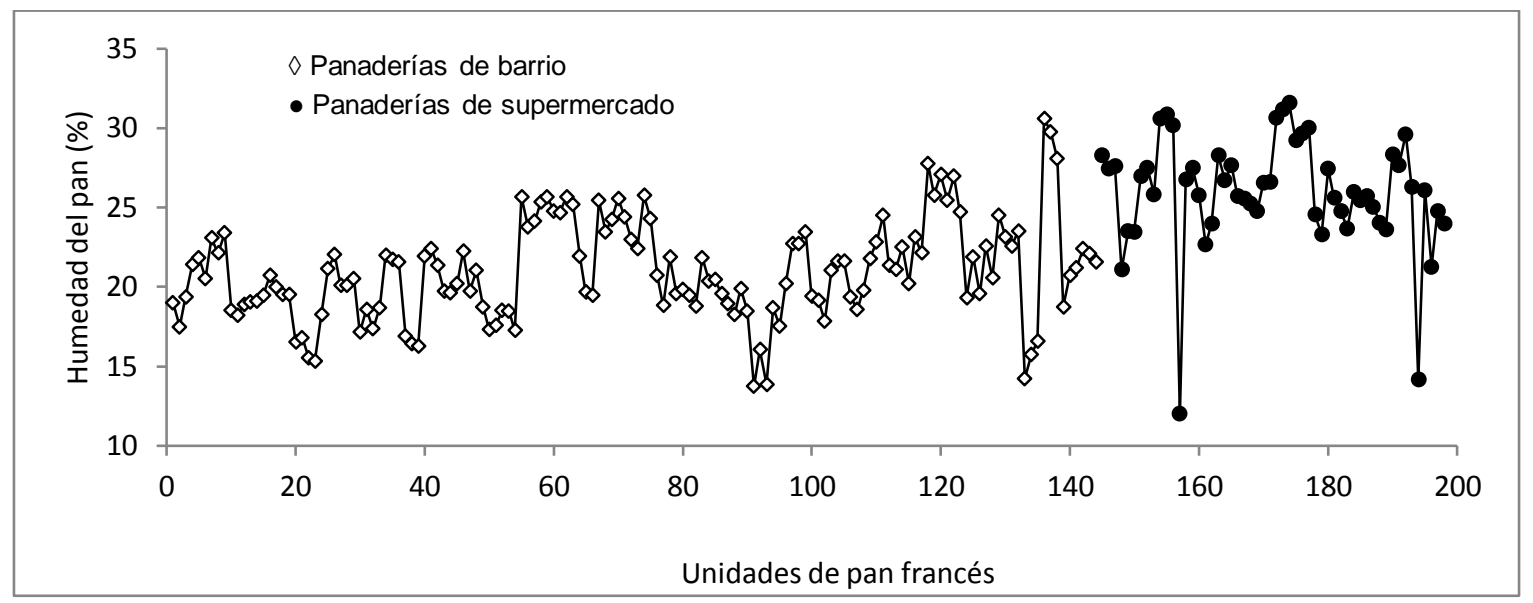

Fig. 3: Porcentaje de humedad en panes francés

\section{Relación y correlación entre variables estudiadas del pan francés}

La Figura 4 muestra una escasa relación causal entre la masa del pan y su volumen; es decir que solo un poco más de la tercera parte de la variación del volumen del pan estaría explicada por la masa $\left(R^{2}=0.3668\right)$. Esta baja relación se debe a que para una misma masa unitaria de pan en gramos existe panes con volúmenes en promedio que van de 5,6 a $9,9 \mathrm{~cm}^{3}$, tal como lo muestra los volúmenes específicos mostrados en la Tabla 1. Esto sería un indicativo de que el volumen del pan no solo depende de la cantidad de masa empleada en su fabricación, sino que presumiblemente el volumen también dependería de otros factores tales como la adición de agentes leudantes y tiempos y temperaturas de fermentación. Además, factores como la técnica o las etapas del mezclado de la masa podrían influir en la calidad del pan principalmente en un aumento del volumen específico (Yin et al., 2021). De esto se deduce que las panaderías evaluadas estarían utilizando distintos parámetros o técnicas para el mezclado de la masa del pan francés que influyen en parámetros como la masa y el volumen.

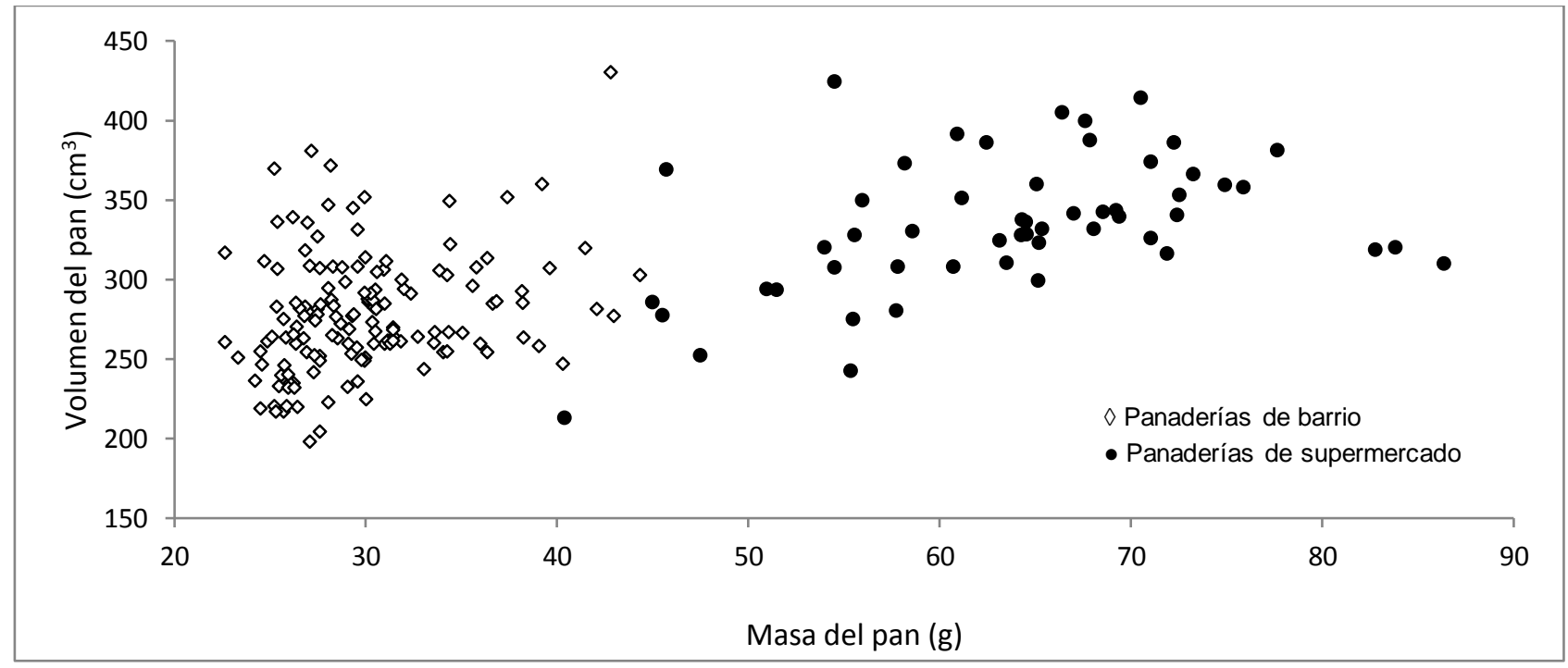

Fig. 4: Relación entre la masa y volumen del pan francés

En cambio, en la Figura 5 se observa que entre la masa del pan y su correspondiente densidad existe una alta correlación ( $\left.R^{2}=0.8599\right)$; es decir, a mayor masa del pan, mayor densidad. El coeficiente de correlación presentado está dentro de lo esperado dada la procedencia de las muestras. Sin embargo, la correlación que debe existir entre la densidad y el volumen del pan es negativa (resultados no mostrados). Según la definición de densidad, se cumple que a mayor volumen menor densidad. Y aunque esta aseveración no se rechaza, sin embargo, la correlación entre estas dos variables es muy baja $\left(R^{2}=0.1279\right)$. 
En la Figura 6, se observa una relación lineal directamente proporcional entre la masa del pan en base húmeda y la masa seca del pan. La masa seca del pan es aquella que queda después de que toda el agua que contiene dicho pan fue retirada por deshidratación en una estufa. Según la Figura 6, esta masa remanente es directamente proporcional a la masa del pan en base húmeda $\left(R^{2}=0.9906\right)$. Este alto grado de correlación entre estas variables podría darnos un indicio de la similitud en las recetas industriales, con la utilización de proporciones diferentes de los ingredientes, el agua retenida en el pan también variaría en concordancia; sobre todo sabiendo que algunos componentes retienen más agua que otros. Las variaciones observadas en la Figura 6 vienen explicadas por la naturaleza misma de las muestras recogidas, al ser éstas de diferentes panaderías y fabricadas bajo diferentes parámetros de proceso y recetas industriales.

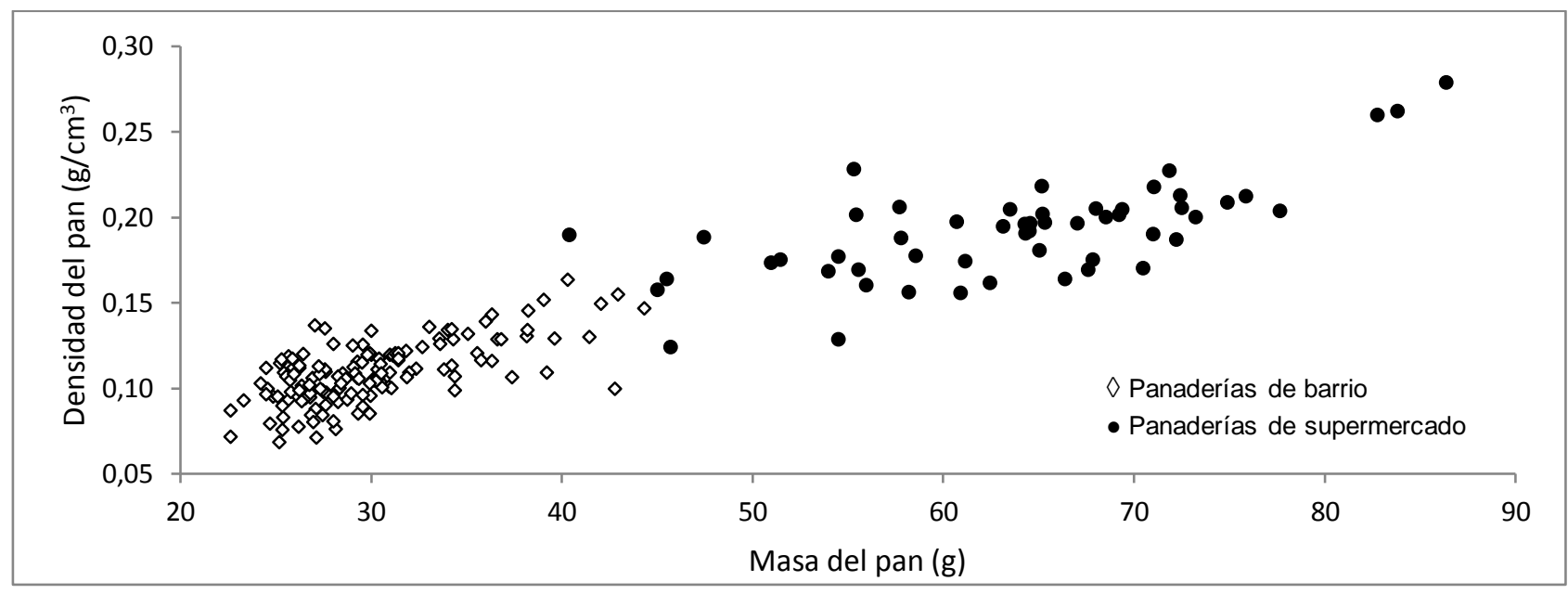

Fig. 5: Relación entre la masa y la densidad del pan francés

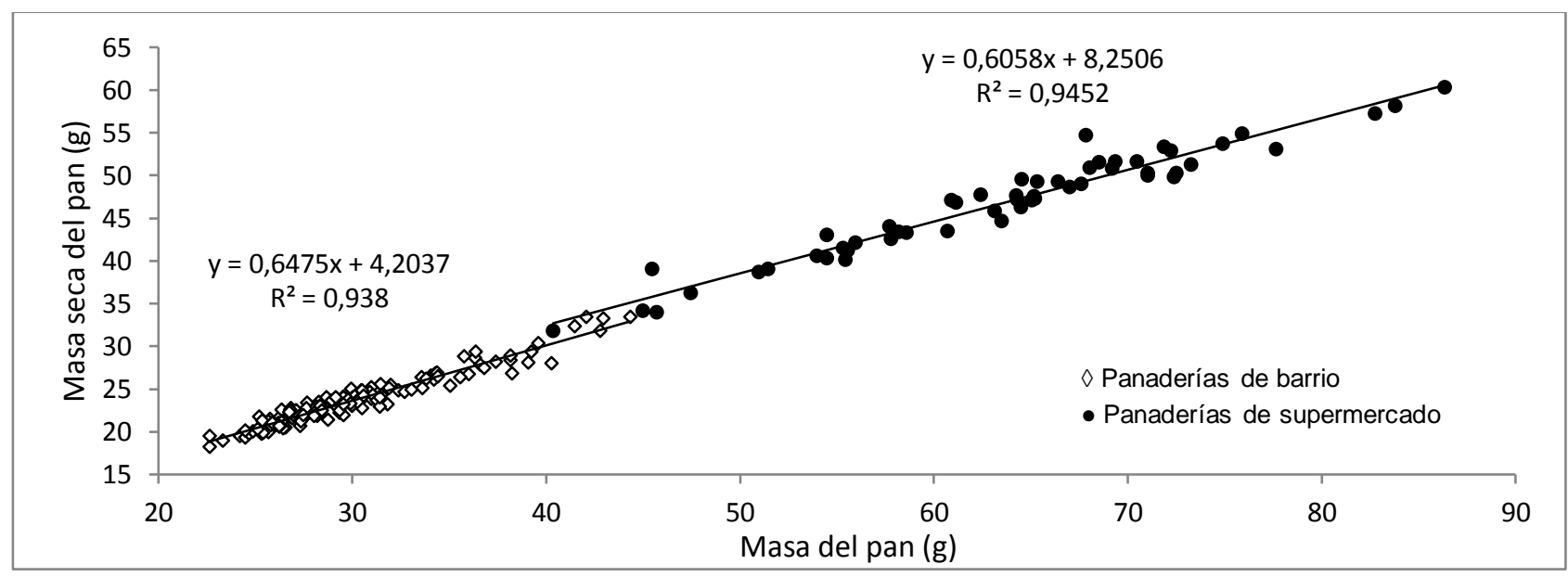

Fig. 6: Relación entre la masa del pan francés en base húmeda y en base seca.

\section{CONCLUSIONES}

Los panes francés fabricados en las panaderías de Lima Metropolitana presentan en forma global una gran variabilidad en sus masas, volúmenes y volúmenes específicos, conforme al análisis de conglomerados y discriminante empleados en el presente trabajo. Los panes de supermercado cumplen con las normas técnicas peruanas respecto al parámetro de humedad. En cambio, los panes de panaderías de barrio poseen un volumen específico muy alto y bajo contenido de humedad. Los panes cumplen con el contenido de sal, sin embargo, presentan baja acidez según lo estipulado en las normas técnicas peruanas. Se deduce que las panaderías de barrio no poseen un proceso estandarizado ni homologado para la fabricación de pan francés. Por lo observado, se recomienda incluir en las normas técnicas el parámetro de volumen específico del pan como requisito de calidad; y que este deberá estar entre $5 \mathrm{~cm}^{3} / \mathrm{g} \mathrm{a} 6 \mathrm{~cm}^{3} / \mathrm{g}$. Además, las panaderías de supermercados deberán disminuir la masa de sus panes, puesto que éstas exceden lo estipulado en las normas correspondientes. 


\section{AGRADECIMIENTOS}

A la Ing. Genny I. Luna Mercado por la revisión completa del trabajo, sus comentarios y acertadas observaciones para la mejora del artículo; a L. M. Soto y T. Silvera por la recolección de las muestras y los análisis de laboratorio.

\section{REFERENCIAS}

AACC International, Method 10-05.01, Guidelines for measurement of volume by rapeseed displacement, 1-4, St. Paul, EUA (2001)

Alvis, A., Pérez, L.J., y Arrazola, G.S., Estudio de propiedades físicas y viscoelásticas de panes elaborados con mezclas de harinas de trigo y de arroz integral, doi: 10.4067/S0718-076420110004000012, Información Tecnológica, 22(4), 107$116(2011)$

Antúnez, L., Giménez, A., y otros tres autores, Consumer perception of salt-reduced breads: Comparison of single and two-bites evaluation, https://doi:10.1016/j.foodres.2017.07.014, Food Research International, 100, 254-259 (2017)

AOAC, Method 983.14. Chloride in cheese, 1-2, Gaithersburg, EUA (2005)

Baardseth, P., Kvaal, K., y otros tres autores, The effects of bread making process and wheat quality on French baquettes, https://doi.org/10.1006/jcrs.2000.0320, J. Cereal Sci., 32, 73 - 87 (2000)

Cardone, G., Grassi, S., Scipioni, A., y Marti, A., Bread-making performance of durum wheat as affected by sprouting, https://doi.org/10.1016/j.Iwt.2020.110021, LWT - Food Sci. Technol., 134, 110021 (2020)

da Silva Torres, E. A. F., Garbelotti, M. L., y Neto, J. M. M., The application of hierarchical clusters analysis to the study of the composition of foods, https://doi.org/10.1016/j.foodchem.2005.08.032, Food Chemistry, 99, 622-629 (2006)

De Bondt, Y.D.E., Hermans, W., Moldenaers, P., y Courtin, C.M., Selective modification of wheat bran affects its impact on gluten-starch dough rheology, microstructure and bread volume, https://doi.org/10.1016/j.foodhyd.2020.106348, Food Hydrocolloids, 113, 106348 (2021)

De La Hoz, E., y Polo, L. L., Aplicación de técnicas de análisis de conglomerados y redes neuronales artificiales en la evaluación del potencial exportador de una empresa, https://doi.org/10.4067/S0718-07642017000400009, Información Tecnológica, 28(4), 67-74 (2017)

Gerardo-Rodríguez, J. E., Ramírez-Wong, B., y otros cinco autores, Effect of part-baking time, freezing rate and storage time on part-baked bread quality, https://doi.org/10.1590/fst.06820, Food Sci. Technol., 41(Suppl. 1), 352-359 (2021)

Gusmão, T. A. S., Alexandre, A. M., y otros cuatro autores, Partial replacement of sodium chloride by potassium chloride in the formulation of French bread: Effect on the physical, physicochemical and sensory parameters, https://doi.org/10.1590/1678-457X.32216, Food Sci. Technol., 37 (Suppl. 1), 55-62 (2017)

$\mathrm{He}, \mathrm{Y} ., \mathrm{y}$ Lu, Q., Increment kinetics of acidity in French bread and critical control point analysis during baking and storage, https://doi:10.1080/10942912.2014.1001070, International Journal of Food Properties, 18(12), 2614$2623(2015)$

Laurent, J., Timmermans, E., y otros tres autores, Variability in yeast invertase activity determines the extent of fructan hydrolysis during wheat dough fermentation and final FODMAP levels in bread,

https://doi:10.1016/j.jifoodmicro.2020.108648, Int. J. Food Microbiol., 326, 108648 (2020)

Melis, S., Verbauwhede, B. C., y otros tres autores, Do puroindolines affect the impact of enzymatic lipid hydrolysis on loaf volume in bread making?, https://doi.org/10.1016/j.foodchem.2019.125273, Food Chemistry, 301, 125273 (2019)

Mohammadi-Moghaddam, T., y Firoozzare, A., Investigating the effectt of sensory properties of black peel marmalade on consumers acceptance by Discriminant Analysis, https://doi.org/10.1016/j.fochx.2021.100126, Food Chemistry: X, 11, 100126 (2021)

Mondal, A., y Datta, A. K., Bread baking - a review, https://doi.org/10.1016/j.jfoodeng.2007.11.014, J Food Eng., 86, 465-474 (2008)

Mudgil, D., Barak, S., y Khatkar, B.S., Optimization of bread firmness, specific loaf volume and sensory acceptability of bread with soluble fiber and different water levels, https://doi:10.1016/j.jcs.2016.06.009, J. Cereal Sci., 70, 186-191 (2016)

NTP 106.004, Panadería, pastelería y galletería. Pan francés. Requisitos, 5-15, Lima, Perú (2016)

NTP 206.006, Productos de panadería. Extracción y preparación de la muestra para el laboratorio, 2-3, Lima, Perú (2016)

NTP 206.008, Productos de panadería. Determinación del porcentaje de acidez titulable, 1-3, Lima, Perú, (2011)

Ortolan, F., Brites, L. T. G., y otros seis autores, Effect of extruded wheat flour and pre-gelatinized cassava starch on process and quality parameters of French-type bread elaborated from frozen dough,

https://doi:10.1016/j.foodres.2015.07.010, Food Res. Int., 76, 402-409 (2015) 
Parenti, O., Guerrini, L., y otros tres autores, Breadmaking with an old wholewheat flour: Optimization of ingredients to improve bread quality, https://doi.org/10.1016/j.Iwt.2019.108980, LWT - Food Sci. Technol., 121, 108980 (2020)

Pérez, C., Análisis multivariante de datos, $1^{\text {a }}$ ed., 323 - 424, Grupo Editorial Garceta, ISBN: 978-84-1545-273-7, Madrid, España (2013)

Rodrigues-Ferreira, S.M., De Oliveira, P.V., y Pretto, D., Parâmetros de qualidade do pão Francés, https://doi:10.5380/cep.v19i2.1240, Bol Centro Pesquisa Processamento Aliment,19(2), 301-318 (2001)

Rossmann, A., Scherf, K. A., y otros tres autores, Effects of a late $\mathrm{N}$ fertiliser dose on storage protein composition and bread volume of two wheat varieties differing in quality, https://doi.org/10.1016/j.jcs.2020.102944, J. Cereal Sci., 93, 102944 (2020)

Scanlon, M. G., y Zghal, M. C., Bread properties and crumb structure, https://doi.org/10.1016/S0963-9969(01)00109-0, Food Res. Int., 34, 841-864 (2001)

SNI (Sociedad Nacional de Industrias), Instituto de Estudios Económicos y Sociales, Perú, Reporte Sectorial № 072018, Elaboración de Productos de Panadería, (www.sni.org.pe) (2018)

Svec, I., y Hrusková, M., Evaluation of wheat bread features, https://doi.org/10.1016/j.jfoodeng.2009.09.022, J. Food Eng., 99, 505-510 (2010)

Yin, J., Cheng, L., y otros cinco autores, Use of two-stage dough mixing process in improving water distribution of dough and qualities of bread made from wheat-potato flour, https:// doi:10.1016/s2095-3119(20)63433-5, J. Integrative Agriculture, 20(1), 300-310 (2021)

Yousaf, A. A., Abbasi, K. S., y otros cinco autores, Physico-chemical and nutraceutical characterization of selected indigenous guava (Psidium guajava L.) cultivars, https://doi.org/10.1590/fst.35319, Food Sci. Technol, Campinas, 41(1), 47-58 (2021) 
